\title{
PI7-20. Lentiviral vector-based vaccine against SIV infection and simian AIDS
}

\author{
A Beignon*, K Mollier, C Liard, S Munier, J Riviere, F Coutant, A Boulay, \\ L Caleechurn, P Souque, C Bauche and P Charneau
}

Address: CNRS-Institut Pasteur, Paris, France

* Corresponding author

from AIDS Vaccine 2009

Paris, France. 19-22 October 2009

Published: 22 October 2009

Retrovirology 2009, 6(Suppl 3):P302 doi: I0.I I86/I 742-4690-6-S3-P302

This abstract is available from: http://www.retrovirology.com/content/6/S3/P302

(C) 2009 Beignon et al; licensee BioMed Central Ltd.

\section{Background}

AIDS vaccination has a pressing need for more potent vaccination vectors capable of eliciting strong, diversified and long-lasting cellular immune responses against HIV. Lentiviral vectors have a proven efficiency not only as gene delivery vehicles for gene therapy applications but also as vaccination tools. This is likely due to their ability to transduce non-dividing cells, including dendritic cells, enabling a sustained endogenous antigen presentation and thus the induction of high proportions of specific cytotoxic T cells and long-lasting memory T cells.

\section{Methods}

The protective efficacy of a lentiviral vector based vaccine was assessed in the SIVmac251/cynomolgus macaques model.

\section{Results}

Our prime-boost vaccination strategy using lentiviral vectors pseudotyped with a glycoprotein G from two noncross-reactive VSV serotypes elicited robust and broad cellular immune responses against the vector-encoded antigen, SIV GAG. Vaccination conferred strong protection against a massive intra-rectal challenge with SIVmac251 as evidenced both by the reduction of viremia at the peak of primo-infection (a mean of over $2 \log 10$ fold reduction) and the full preservation of the central memory CD $4+\mathrm{T}$ cells during the acute phase. Although vaccinees continued to display lower viremia than control macaques during the early chronic phase, these differ- ences were not statistically significant by day 50 post-challenge. This encouraging pilot prophylactic trial gave the proof-of concept for the strong potential of the lentiviral vector system for vaccination. Various optimizations were successfully tested in mice to increase the immunogenicity of our vaccine. An improved vaccine - with a codon-optimized sequence encoding 2 viral antigens, GAG together with NEF, under the control of a promoter devoid of associated enhancer activity and injected via the intramuscular route - is being evaluated in a therapeutic setting, in viremic rhesus monkeys.

\section{Conclusion}

Gene transfer vectors derived from HIV-1 appear as promising candidates for vaccination against HIV-1 infection. 